\title{
Transtornos mentais: detecção e prevenção na criança e no adolescente*
}

\author{
Um parecer coletivo do IN SERM
}

Uma em cada oito crianças, na França, sofre de algum transtorno mental. Que se trate de autismo, de hiperatividade, de transtornos obsessivo-compulsivos, de transtornos do humor, de ansiedade, de anorexia, de bulimia ou de esquizofrenia, esses transtornos podem ter uma repercussão considerável sobre o devir da criança. No entanto, passam-se freqüentemente vários anos entre o aparecimento dos primeiros sintomas e sua detecção. Pais, professores, educadores e médicos devem ser mobilizados para reduzir esse tempo de latência. Para isso eles precisam ser mais bem informados e preparados para o uso de instrumentos de diagnóstico resultantes dos últimos avanços realizados por pesquisas.

Pela primeira vez na França, um documento agrupa o conjunto dos dados científicos e médicos existentes sobre os distúrbios mentais da criança e do adolescente. Especialistas de várias disciplinas foram convocados pelo Instituto Nacional de Saúde e de Pesquisa Médica (INSERM), a pedido da Canam (Caisse nationale d'assurance maladie des travailleurs indépendants), ${ }^{1}$ para analisar as publicações internacionais sobre

* Trata-se do dossiê de imprensa divulgado pelo INSERM (Instituto Nacional Francês de Pesquisa Médica), em 6 de fevereiro de 2003, e divulgado também na página do INSERM na internet (endereço http://.inserm.fr/). Tradução de Regina Orth de Aragão.

1. Caixa nacional de seguro de saúde dos trabalhadores independentes. 
o assunto. A avaliação coletiva resultante mostra a importância da detecção precoce dos problemas psiquiátricos. Ela sublinha igualmente os progressos recentes dos conhecimentos a respeito de seus modos de manifestação.

Várias recomendações foram propostas pelos especialistas ao final desse trabalho, entre as quais destacamos as seguintes:

- Uma boa informação dos pais, professores e educadores. Em contato com a criança, eles são os mais aptos a perceber os primeiros sinais de um distúrbio mental.

- Uma melhor formação dos clínicos gerais, dos pediatras, dos médicos escolares e da $\mathrm{PMI}^{2}$ para a detecção dos distúrbios mentais, levando a uma orientação mais rápida para as estruturas especializadas.

- Uma aceleração da transferência dos últimos resultados obtidos em pesquisas para a prática clínica dos psiquiatras infantis.

- Uma melhoria do dispositivo existente de vigilância sistemática, com instrumentos de detecção mais eficazes.

- Um acompanhamento especial das crianças “à risco”, mais suscetíveis de virem a desenvolver um distúrbio mental (crianças de pais sofrendo de distúrbios mentais, crianças prematuras...)

- O estudo e a adaptação de programas de prevenção ao conjunto das crianças, àquelas suscetíveis de desenvolver um distúrbio e às crianças e aos adolescentes que já apresentem sinais ou sintomas.

- O prosseguimento das pesquisas sobre os mecanismos de aparição dos distúrbios mentais, sobre o impacto dos diferentes fatores de risco que os condicionam e sobre o valor de predição de certos sinais precoces, para a construção de novos instrumentos de detecção.

Segundo a Organização Mundial de Saúde (OMS), o peso dos distúrbios mentais no conjunto das doenças deve aumentar em torno de 50\% em 2020. Esses distúrbios se tornariam então uma das cinco principais causas de morbidez na criança. (O relatório OMS 2001 prevê que a carga de morbidade imputável aos distúrbios mentais passará de 10\% em 1990 para 15\% em 2020).

\section{Uma em cada oito crianças, na França, sofre de transtornos mentais}

Segundo as investigações francesas e internacionais, em torno de $12 \%$ das crianças e adolescentes seriam afetados por um ou vários distúrbios mentais.

2. Protection Materno-Infantile (Serviços de Proteção Materno-Infantil), que fazem parte do equipamento básico de saúde na França, presentes em todos os bairros das grandes cidades e também nos pequenos municípios (N. da T.). 
Algumas dessas desordens manifestam-se especificamente nesse período da vida - autismo, hiperatividade, anorexia mental, transtornos das condutas; outros são também encontrados nos adultos - esquizofrenia, transtornos ansiosos, transtornos do humor. Todos foram levados em consideração nessa avaliação coletiva, com exceção dos distúrbios da conduta que farão objeto de uma próxima avaliação.

Na França, todos os dados epidemiológicos existentes sobre os transtornos mentais na criança e no adolescente concordam com as estimativas internacionais (estudos americanos, europeus, da Ásia e da Nova Zelândia). Com efeito, apesar da variedade dos ambientes encontrados, a prevalência e a morbidez psiquiátrica são elevadas de um país para outro. O que suscita numerosas interrogações.

\section{Alguns números:}

$5 \%$ das crianças sofrem de transtornos ansiosos, e 1 a $2 \%$ são hiperativas. $\mathrm{Na}$ adolescência, os transtornos do humor (transtornos depressivos e transtornos maníaco-depressivos...) aumentam porque atingem 3\% dos jovens entre 13-19 anos. A bulimia concerne $1 \%$ das jovens de 17-19 anos. E a anorexia afeta 0,2\% das adolescentes de 15-19 anos, se considerarmos somente as formas de anorexia restritiva pura. Quanto ao autismo e à esquizofrenia, são doenças mais raras, que atingem cerca de $1 \%$ das crianças e dos adolescentes.

\section{Esperas muito longas para os diagnósticos, quaisquer que sejam os transtornos}

Os transtornos mentais têm uma repercussão considerável sobre o desenvolvimento da criança e sobre o sucesso de suas aprendizagens. No entanto, o diagnóstico freqüentemente só é estabelecido muito tempo depois do aparecimento dos primeiros sintomas, porque ele é difícil de ser proposto para uma criança que está, por definição, em pleno desenvolvimento. Classificações das doenças, entre as quais a Classificação Internacional das Doenças (CID 10) e o DSM (Diagnostic and Statistical Manual por Mental Diseases), as mais utilizadas, propõem critérios para facilitar esse diagnóstico. Essas classificações evoluem em função do avanço dos conhecimentos científicos e médicos.

O autismo infantil é um distúrbio severo do desenvolvimento que atinge as funções de comunicação e de linguagem, o desenvolvimento das relações sociais e a imaginação. Para limitar a evolução desses déficits, um diagnóstico precoce é indispensável. Com efeito, um acompanhamento pelos programas educativos 


\section{$\begin{array}{lllllll}R & E & V & I & S & T & A\end{array}$ \\ LATINOAMERICANA \\ DE PSICOPATOLOGIA \\ F U N D A M E T T L \\ ano VIII, n. 3, set/2005}

intensos antes da idade de 4 anos permite obter melhoras ao nível da linguagem e do desenvolvimento cognitivo. Mais comumente o diagnóstico é dado por volta dos 3-4 anos, e às vezes mais tarde. Mas a maioria dos pais percebe os primeiros sintomas de um desenvolvimento anormal durante os dois primeiros anos da vida de sua criança.

O grupo de especialistas lembra que as crianças que apresentam um atraso de linguagem, uma ecolalia (repetição de palavras), ou um retraimento sobre si mesmas devem ser levadas a centros de diagnóstico para beneficiarem-se de uma avaliação completa (cognitiva, neurológica, metabólica, por imageamento cerebral...).

A hiperatividade manifesta-se na criança por três sintomas principais: uma agitação incessante, um déficit de atenção e uma impulsividade que leva a interromper as atividades de outras pessoas. Essas diversas manifestações repercutem sobre o funcionamento da criança, na escola, em casa e durante as atividades de lazer.

Segundo os especialistas, o diagnóstico, em geral proposto em torno de 7 anos de idade, após o aparecimento das primeiras dificuldades de aprendizagem escolar devidas à agitação e à falta de concentração da criança, poderia ser dado mais cedo, desde os 3-4 anos. Alguns sinais de apelo, como a agitação ou a intolerância à frustração, aparecem em geral bem antes da entrada na escola primária. Os especialistas lembram que o diagnóstico precoce é fundamentalmente necessário já que existem tratamentos eficazes, e que a hiperatividade está associada, em 50 a 70 \% dos casos, a outros distúrbios mentais (distúrbios de conduta, distúrbios ansiosos depressivos, distúrbios de aprendizagem, tics...).

Os transtornos obsessivo-compulsivos (ou TOC) aparecem por volta dos 10 anos de idade e se parecem com as formas adultas dessa doença. As obsessões relacionam-se com a limpeza, o medo de catástrofes, os temas sexuais ou religiosos. E as compulsões manifestam-se pela repetição anormal e exagerada dos mesmos gestos - rituais de lavagem e de verificação - sobretudo em certos períodos do dia (ao deitar-se, no banho, nas refeições etc.).

Os especialistas explicam o diagnóstico tardio desses transtornos, em primeiro lugar pela dificuldade em distinguir os comportamentos obsessivocompulsivos dos rituais de desenvolvimento, freqüentes na criança; em seguida, pelo caráter muito pouco específico dos sinais de apelo, tais como a lentidão ou as dificuldades escolares. O grupo de especialistas recomenda uma abordagem diagnóstica que deva permitir a pesquisa de outros transtornos mentais, associados ao TOC em 60 a 70\% dos casos (transtornos depressivos, tics).

Existem na criança diversos transtornos ansiosos que se somam freqüentemente uns aos outros durante o desenvolvimento. O transtorno ansiedade de separação é o mais freqüente: ele começa por volta dos 6-7 anos e 
se traduz por um desespero intenso da criança quando ela é separada das pessoas às quais está ligada. A hiperansiedade, por sua vez, começa por volta dos 8-9 anos. Ela se manifesta por preocupações excessivas relativas aos resultados escolares, com sintomas de agitação, de fadiga, perturbações do sono e da memória. Quanto aos ataques de pânico, eles podem levar, se se repetirem, ao transtorno de pânico por volta dos 15-19 anos. Em geral, entre os 6 e 12 anos exprimem-se as fobias simples - medo de um objeto ou de uma situação - depois, entre 12 e 15 anos, as fobias sociais - medo do contato com o outro, inclusive com outras crianças - gerando um sentimento de sofrimento intenso. O estado de estresse póstraumático, um transtorno ansioso, foi durante muito tempo ignorado na criança, mesmo pelos profissionais de saúde. No entanto, suas conseqüências (transtornos do sono, acessos de raiva, alterações do desempenho escolar, auto-agressividade, hiper-vigilância) sobre a vida da criança são importantes: em 50\% dos casos, o transtorno persiste para além de 12 meses após o acontecimento; ele pode inclusive se perenizar.

Os especialistas constatam que freqüentemente é o surgimento de complicações tais como a recusa escolar, os distúrbios da adaptação social ou do comportamento, as tentativas de suicídio, ou os distúrbios depressivos que levam, tardiamente, ao diagnóstico dos transtornos ansiosos na criança.

A repercussão psicossocial e a evolução por vezes crônica dos transtornos do humor (distimia, transtorno depressivo maior, transtorno maníaco-depressivo) na criança e no adolescente requerem o diagnóstico precoce e um tratamento adequado. De acordo com os especialistas, eles aparecem mais freqüentemente nos adolescentes, principalmente nas meninas, precedidos por transtornos ansiosos e do comportamento (hiperatividade). As manifestações desses transtornos do humor e as tentativas de suicídio (em $40 \%$ das crianças e dos adolescentes deprimidos, segundo os trabalhos americanos) são sinais de alerta importantes para realizar um diagnóstico.

Os transtornos do comportamento alimentar (TCA) atingem sobretudo as adolescentes que têm tendência a se focalizar sobre a imagem de seus corpos. Com efeito, as futuras anoréxicas ou bulímicas vivem com maiores dificuldades do que outras adolescentes o período pubertário, momento-chave da maturação física e psíquica. O diagnóstico e o tratamento intervêm tardiamente, sobretudo para as anoréxicas, porque as famílias não vêem imediatamente a gravidade da situação. Bruscas variações brutais de peso, uma perda de peso de pelo menos $10 \%$, uma amenorréia (ausência de regras), preocupações excessivas em torno da imagem do corpo, da alimentação e uma baixa auto-estima devem, segundo os especialistas, ser consideradas sinais de um comportamento anoréxico ou bulímico. Esses sinais devem levar rapidamente à consulta médica. 


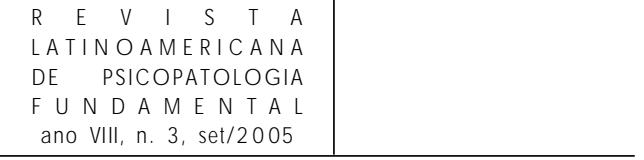

A esquizofrenia é caracterizada por transtornos do curso do pensamento, por idéias delirantes, por alucinações, uma discordância afetiva. Muito rara na criança, ela começa geralmente no final da adolescência ou no início da idade adulta, como se a doença ficasse silenciosa no plano clínico antes desse período. As primeiras manifestações parecem ligadas aos remanejamentos cerebrais que acompanham a passagem para a idade adulta. Vários estudos interessaram-se pela natureza dos sintomas que surgem durante o ano precedendo o primeiro episódio psicótico. Se os cientistas observam freqüentemente um retraimento social, constatam também distúrbios do sono, uma certa irritabilidade, idéias suicidas, ou, ainda, uma baixa da atenção. Os especialistas notam, no entanto, que esses sintomas são excessivamente pouco específicos para orientar um diagnóstico precoce.

\section{Para perceber os sinais de alerta, sensibilizar o meio, formar os médicos}

Os pais, professores e educadores que convivem com a criança no cotidiano são os mais aptos a perceber uma anomalia no seu desenvolvimento ou no seu comportamento. Além da mídia, que contribui para essa sensibilização, os jovens pais poderiam também recorrer a um site institucional de informação da internet sobre essas questões, recomendado pelos especialistas. O grupo de especialistas preconiza também a utilização de um questionário durante as visitas ao médico ou ao pediatra, para que os pais indiquem com maior precisão suas observações sobre o comportamento da criança desde a visita anterior.

Para uma melhor detecção, é indicado também desenvolver a formação dos médicos de primeira linha (clínicos gerais, pediatras, médicos escolares ou de PMI). Freqüentemente em contato com as crianças, eles devem ser capazes de reconhecer os sinais [de apelo] de um distúrbio, para encaminhar a criança a um especialista ou a um centro de diagnóstico. Considerando a freqüência dos transtornos psiquiátricos na população geral, os especialistas do Inserm estimam que esses profissionais da saúde deveriam beneficiar-se durante seus estudos médicos de uma formação sobre o desenvolvimento cognitivo-sócio-afetivo das crianças e de um ensinamento sobre os transtornos mentais. Além disso, eles devem poder dispor de um tempo suficiente para esse tipo de consulta, que deveria assim ser reconhecida como um ato médico indiscutível, inscrito na nomenclatura. Um diagnóstico tardio é freqüentemente prejudicial para a evolução dos transtornos mentais. Portanto, é importante dar aos psiquiatras infantis os meios de diagnosticar o mais precocemente possível esses distúrbios. Para isso, o grupo 
de especialistas recomenda que os profissionais beneficiem-se dos novos avanços científicos e médicos para uma difusão e um ensino dos conhecimentos adquiridos, em particular nos campos do desenvolvimento cerebral, das ciências cognitivas e das imagens cerebrais por ressonância magnética funcional. O que permitiria integrar rapidamente esses conhecimentos às suas práticas clínicas.

\section{Detectar graças aos dispositivos de saúde existentes}

Na França, a detecção dos transtornos mentais deve se apoiar no dispositivo existente de acompanhamento sistemático das crianças, do nascimento até a adolescência.

Os exames dos dois primeiros anos (no nascimento, no quarto, nono e vigésimo-quarto mês) são importantes para avaliar o desenvolvimento da criança. Também um instrumento permitindo estabelecer um índice individual de desenvolvimento poderia substituir com vantagem o que é atualmente empregado, fundado sobre a comparação das crianças a uma média. O grupo de especialistas recomenda então integrar os indicadores do desenvolvimento sensório-motor, cognitivo e emocional às rubricas do caderno de saúde dos dois primeiros anos, e, a partir do nono mês, uma avaliação da atenção e da memória.

Os especialistas sugerem igualmente que um questionário como o CHAT (Checklist for autism in toddlers) ${ }^{3}$ destinado às crianças de 1 a 2 anos seja desenvolvido. Esse instrumento que interroga sobre o jogo social, o jogo simbólico, o apontamento protodeclarativo (o fato de mostrar com o dedo os objetos interessantes para o bebê) ou ainda a atenção conjunta, permitiria, segundo o grupo de especialistas, detectar o autismo a partir dos 18 meses.

Autismo: os sinais que devem alertar

Mesmo se não são exclusivamente específicos do autismo, alguns déficits devem alertar os médicos, e desencadear um exame secundário por um psiquiatra infantil.

- Ausência de balbucio aos 12 meses;

- ausência de gesto de tipo apontar ou tchau com a mão aos 12 meses;

- ausência de palavra aos 16 meses;

- ausência de combinação espontânea de duas palavras aos 24 meses;

- qualquer perda de competência (de linguagem ou social) em qualquer idade.

3. Em inglês no texto original (N. da T.). 


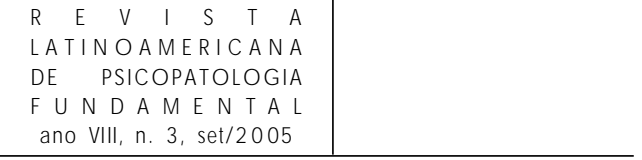

Para os especialistas seria conveniente, no início da escolarização, isto é, no ingresso na escola maternal, procurar os atrasos de fala, as dificuldades de concentração, os acidentes e as quedas freqüentes que podem ser sinais de hiperatividade. Uma maleta de avaliação do desenvolvimento sob forma de jogo poderia ser proposta aos médicos e aos pediatras, com esse objetivo.

O exame realizado aos 5-6 anos, antes da entrada na escola primária, ${ }^{4}$ é importante por oferecer a oportunidade de reunir pais, professores e médicos. Para dar a ele um conteúdo homogêneo em todos os estabelecimentos escolares, o grupo de especialistas preconiza que se institua uma caderneta, concebida como um instrumento para as enfermeiras, os médicos escolares, os professores e os pais. Ela contribuiria para informá-los sobre os transtornos que podem aparecer nessa idade.

Na pré-adolescência e na adolescência vários transtornos podem aparecer: os transtornos ansiosos, como o transtorno do pânico, os transtornos depressivos ou os transtornos do comportamento alimentar, tais como a anorexia e a bulimia. É, portanto, importante efetuar uma avaliação em todos os estabelecimentos escolares. Uma baixa nos resultados escolares nas quinta ou sexta séries do primeiro grau é, muitas vezes, reveladora de um transtorno ansioso-depressivo. Instrumentos simples de utilização, do tipo questionários, estão sendo validados na França: eles poderiam permitir detectar a anorexia, a bulimia e os transtornos ansioso-depressivos nos adolescentes.

O diagnóstico dos transtornos mentais no contexto das avaliações sistemáticas na população geral deve necessariamente prosseguir com o acompanhamento de um especialista, o único com condições de fazer um diagnóstico e uma avaliação completa.

\section{Acompanhar as crianças de risco e desenvolver programas de prevenção}

Se o papel dos fatores genéticos está bem demonstrado para o surgimento dos transtornos mentais, é preferível falar de suscetibilidade genética, porque os fatores genéticos devem interagir com outros fatores, particularmente os do ambiente, para que se expresse o transtorno.

A prevenção em matéria de transtornos mentais na criança e no adolescente deve levar em conta os dados obtidos pela pesquisa clínica. Mesmo que

4. Na França, o ingresso no ensino fundamental se dá aos 6 anos, correspondendo ao último ano do ciclo de Educação Infantil no Brasil (N. da T.). 
numerosas interrogações persistam, esses dados, no entanto, atualmente permitem enunciar a prevenção segundo três eixos principais:

- a prevenção dita generalizada, que se dirige às crianças e aos adolescentes que não foram selecionados tendo como base um risco definido;

- a prevenção dita seletiva, que visa as crianças e os adolescentes apresentando um risco significativamente mais elevado do que a média de desenvolver um transtorno mental;

- a prevenção dita indicada, que se dirige aos sujeitos que já apresentam sinais ou sintomas de transtorno mental.

Uma criança que sofre de um transtorno mental tem maiores riscos de desenvolver um outro. O grupo de especialistas recomenda que seja acompanhada em centro especializado toda criança apresentando um transtorno, a fim de prevenir o aparecimento de uma patologia adicional.

As crianças tendo pai ou mãe, um irmão, uma irmã ou um outro membro da família apresentando um transtorno mental correm o risco de desenvolver o mesmo transtorno. Esse risco varia, no entanto, em função dos transtornos psiquiátricos e pode refletir determinantes genéticos e/ou do ambiente. $\mathrm{O}$ determinismo genético é sobretudo importante para o autismo, e é por isso que o grupo de especialistas recomenda uma avaliação anual durante o período pré-escolar de toda criança de família que tenha um membro com autismo.

As crianças de famílias apresentando transtornos mentais do tipo alcoolismo, toxicomania, transtornos depressivos, são também suscetíveis de desenvolver uma patologia, que não é necessariamente a mesma de seus pais. O grupo de especialistas recomenda, então, que se efetue acompanhamento das crianças cujos pais são tratados em centros especializados, e que se promovam programas de prevenção permitindo às famílias capacitação em comunicação e resolução de problemas.

O desenvolvimento dos programas de prevenção e sua avaliação em termos de aceitabilidade e de resultados devem ser encorajados no contexto francês, privilegiando tradicionalmente as opções mais individuais. Essas últimas guardam certamente sua importância, mas são mais difíceis de serem aplicadas à população em geral ou fora das redes de tratamento clássicas.

Por outro lado, o grupo de especialistas enfatiza a necessidade de melhorar o acesso aos tratamentos para as crianças e os adolescentes sofrendo de distúrbios mentais clinicamente significativos. Com efeito, os progressos em termos de prevenção conduzem a uma melhor detecção dos distúrbios manifestos ou iniciais, detecção que permanecerá um objetivo inútil se os cuidados adaptados e diversificados não puderem ser propostos dentro dos prazos corretos. 


\section{$\begin{array}{lllllll}R & E & V & I & S & T & A\end{array}$ \\ LATINOAMERICANA \\ DE PSICOPATOLOGIA \\ F U N D A M E T A L \\ ano VIII, n. 3, set/2005}

Desigualdade dos sexos e da idade

O sexo e a idade têm influência sobre a expressão dos transtornos mentais. Com efeito, os mecanismos hormonais não são os mesmos nos meninos e nas meninas, e modificações neurobiológicas distintas intervêm no curso do desenvolvimento cerebral e mental.

Os meninos são mais vulneráveis do que as meninas aos transtornos do desenvolvimento antes da idade de 12-13 anos, mas a situação se inverte na adolescência. As meninas são então mais atingidas, e continuam a sê-lo durante a idade adulta. Por outro lado, os dois sexos não exprimem o mesmo tipo de transtornos: em geral, os meninos sofrem mais de transtornos externalizados (hiperatividade...) e as meninas de transtornos internalizados (transtornos depressivos, transtornos do comportamento alimentar...).

\section{Prosseguir a pesquisa sobre a interação entre os diversos fatores de risco}

Os estudos de agrupamentos 5 são importantes para identificar o impacto dos diferentes fatores genéticos e ambientais, e suas interações no desenvolvimento dos transtornos mentais.

Entre os acontecimentos ambientais que podem ter um impacto sobre o surgimento dos transtornos mentais, é preciso citar os traumatismos que podem ocorrer durante a gravidez e também no parto. Esses dois momentos são realmente períodos críticos, porque o sistema nervoso do bebê está em pleno desenvolvimento. Os mecanismos de ação dos fatores ambientais, sociais e afetivos, durante a gravidez e a primeira infância, devem ainda ser aprofundados.

Para melhor captar o impacto desses fatores sobre o desenvolvimento afetivo e cognitivo da criança e sobre o aparecimento de distúrbios mentais, o grupo de especialistas recomenda que seja acompanhada regularmente uma grande população de crianças, por meio de medidas clínicas, psicopatológicas, biológicas e neuro-cognitivas; integrar nesses estudos a análise dos marcadores genéticos e uma pesquisa sobre as alterações cerebrais eventuais, por imageamento. Esses agrupamentos permitiriam também estudar o valor preditivo de certos sinais

5. Cohorte no original, significando o conjunto de pessoas tendo vivido um mesmo acontecimento demográfico (nascimento, casamento, nascimento do primeiro filho...) durante o mesmo ano ou mesmo período. Tratando-se de pessoas nascidas no mesmo ano, fala-se em geração, e para as pessoas que se casaram no mesmo ano, emprega-se a expressão promoção de casamento (N. da T.). 
precursores durante os primeiros meses de vida para o autismo, as perturbações dos processos de atenção e do controle motor para a hiperatividade, as perturbações observadas no período pré-pubertário para os distúrbios do comportamento alimentar e para a esquizofrenia.

\section{Decifrar os mecanismos de aparecimento: o desafio das pesquisas futuras}

Até hoje, os mecanismos fisiopatológicos dos transtornos mentais não estão claramente elucidados. A fim de compreender melhor como os disfuncionamentos aparecem, o grupo de especialistas recomenda o aprofundamento dos conhecimentos relativos ao desenvolvimento do sistema nervoso da criança. Porque conhecemos mal a maneira pela qual as diferentes funções mentais se organizam. Estudos de caso de lesões cerebrais no adulto conduziram à individualização dos "módulos" funcionais, redes de neurônios intervindo nas diferentes funções mentais (reconhecimento dos rostos, linguagem etc.). Não sabemos se esses módulos desenvolvem-se independentemente uns dos outros ou simultaneamente. Também, na criança, os módulos não estão ainda bem estabelecidos, independentes uns dos outros, e os disfuncionamentos tocam as funções durante sua maturação. No entanto, há casos nos quais os atrasos mentais ou os transtornos de aprendizagem aparecem isoladamente. A questão fica então em suspenso: as manifestações dos transtornos são resultado de um déficit único ou de uma combinação de déficits?

Graças aos progressos das imagens cerebrais por ressonância magnética funcional, podemos observar in vivo anomalias ligadas às diferentes patologias, localizá-las e perceber o momento em que elas surgem. Mas o recrutamento de pacientes psiquiátricos para essas pesquisas é limitado. O trabalho dos pesquisadores poderia ser facilitado pela coordenação dos trabalhos sobre o imageamento cerebral e a criação de bancos de dados de imagens cerebrais.

Por outro lado, a emergência de novos modelos animais, extremamente raros hoje, poderia ser útil para compreendermos melhor os processos pelos quais a patologia se desenvolve. 